\title{
Method of Detection Abnormal Features in Ionosphere Critical Frequency Data on the Basis of Wavelet Transformation and Neural Networks Combination
}

\author{
O. V. Mandrikova ${ }^{1,2}$, Yu. A. Polozov ${ }^{1,2}$, V. V. Bogdanov ${ }^{1}$, E. A. Zhizhikina ${ }^{2}$ \\ ${ }^{1}$ Institute of Cosmophysical Researches and Radio Wave Propagation FEB RAS, Petropavlovsk-Kamchatskij, Russia; ${ }^{2}$ Kamchatka \\ State Technical University, Petropavlovsk-Kamchatskij, Russia. \\ Email: oksanam01@mail.kamchatka.ru, up_agent@mail.ru, vbogd@ikir.ru
}

Received 2012.

\begin{abstract}
The research is focused on the development of automatic detection method of abnormal features, that occur in recorded time series of ionosphere critical frequency $\mathrm{f}_{\mathrm{O}} \mathrm{F} 2$ during periods of high solar or seismic activity. The method is based on joint application of wavelet-transformation and neural networks. On the basis of wavelet transformation algorithms for the detection of features and estimation of their parameters were developed. Detection and analysis of characteristic components of time series are performed on the basis of joint application of wavelet transformation and neural networks. Method's approbation is performed on $\mathrm{f}_{\mathrm{O}} \mathrm{F} 2$ data obtained at the observatory "Paratunka" (Paratunka settlement, Kamchatskiy Kray).
\end{abstract}

Keywords: Wavelet Transformation; Neural Networks; Critical Frequency of Ionosphere; Abnormalities; Earthquakes

\section{Introduction}

The subject of the investigationsis recorded time series of ionospheric parameters, which include components with different internal structure and determined by density of atmosphere, its chemical compound and the spectral characteristics of solar radiation [1,2]. Ionosphere research is carried out by distant methods, one of which is vertical radio probing. Frequency of carrier radio impulse for which the given area of ionosphere becomes transparent, is called critical $\left(\mathrm{f}_{\mathrm{O}} \mathrm{F} 2\right)$ and it characterises electron concentration.

Against the regular changes caused by a daily and seasonal course, abnormal features with duration from some tens minutes till several hours [2-13] are observed in the $\mathrm{f}_{\mathrm{O}} \mathrm{F} 2$ data. These anomalies have various structure and arise against powerful ionospheric disturbances which are caused by solar activity, in seismoactive areas they can arise during the seismic activity increase periods [2-13]. Complex structure of the ionospheric data makes traditional methods of the time series analysis inefficient for their analysis and abnormalities detection, because these methods are based on the procedure of smoothing and lead the important information loss [2,5]. Main tools for abnormalities detection are based on the analysis of the average and median values that does not allow to find out internal dependences in the data and single abnormal features.

In connection with the wide variety of basis functions with compact carriers, wavelet-transformation is an effective tool for complex time series analysis [3,4,1419]. Using the discrete wavelet-transformation construction, the algorithm allowing to allocate abnormal features and to define their parametres in $\mathrm{f}_{\mathrm{O}} \mathrm{F} 2$ data in an automatic mode is offered in this paper.

For characteristic components of $\mathrm{f}_{\mathrm{O}} \mathrm{F} 2$ time series detection and analysis this paper proposes the method based on joint application of wavelet-transformation with neural networks. Neural networks have well proved in complex nonlinear dependences reproduction [6,21-23]. The efficiency of this mathematical tool application for ionospheric data processing and analysis is demonstrated in $[6,11,12,22,23]$. These authors offer ways of $f_{0} F 2$ data analysis and prognosis on the basis of neural networks and show that in many respects their work result is defined by properties of training set. Experimental search 
of suitable training set and neural network architecture is carried out in $[22,23]$. If modelled data are complex and noisy it is necessary to perform their preprocessing and to solve problems of uninformative and redundant data $[5,6,13]$. In $[6,13]$ offered ways of joint application of wavelet-transformation and neural networks for uninformative data removal, developed algorithms of training set formation on the basis of a wavelet-filtration, and showed that the given approach allows to optimise process of network training and to increase length of data anticipation interval. This paper, where the method of $\mathrm{f}_{\mathrm{O}} \mathrm{F} 2$ characteristic components detection and prognosis on the basis of wavelet-packages construction_and neural networks joint application is developed, is continuation of these investigations.

In process of proposed method approbation, abnormal features in $f_{0} F 2$ data, arising during the periods of increased solar activity or caused by processes in lithosphere (seismic events of a power class with $\mathrm{k}>12$ analysis) were detected.

\section{Method description}

Detection of abnormal features and their parametres definition on the basis of discrete wavelet-transforma-

tion. Formally complex time series can be presented as sum of different-scale components with various internal structure [5] $f(t)=\sum_{j} f_{j}(t)$, where $j$ is scale.

As the $f_{j}$ components structure is subject to change in random time moments, the most effective way for their description is application of approximation methods, based on decomposition of function on basis. Considering analyzed features local character, their different-scale and forms variety, the most suitable space for their representation is wavelet-space $[5,13,14]$.

On the basis of discrete wavelet-transformation for $f_{j}$ components the following representation in the form of the wavelet-scheme is obtained [14, 23]:

$$
f_{j}(t)=\sum_{n} c_{j, n} \Psi_{j, n}(t)
$$

where $\left\{\Psi_{j, n}\right\}_{(j, n) \in Z^{2}}$ is orthonormalized basis of the $L^{2}(R) \quad$ Lebega space , $\Psi_{j, n}=2^{j / 2} \Psi\left(2^{j} t-n\right)$, $f_{j} \in L^{2}(R) . \quad \bar{c}_{j}=\left\{c_{j, n}\right\}_{n \in Z}$ coefficients are result of mapping of $f$ into the space with resolution $j$, $c_{j, n}=\left\langle f, \Psi_{j, n}\right\rangle$.

Without breaking general coherence, we will consider that an initial discrete time series belong to space with scale $j=0$. The importance of representation $f$ as Equation (1) is defined by sorting and storing of different-scale components of complex time series in various spaces $W_{j}$ with resolution $j$ :

$$
W_{j=0}=\oplus_{j=-1}^{-J} W_{j},\left\{\Psi_{j, n}\right\}_{n \in Z} \text { is basis of } W_{j} \text { space. }
$$

For the purpose of possibility to construct adaptive approximating wavelet-schemes, we will use nonlinear mappings [5, 14]:

$$
f_{M}(t)=\sum_{(j, n) \in I_{M}} c_{j, n} \Psi_{j, n}(t),
$$

where $f_{M}$ is projection of $f$ onto $M$ vectors which indexes are contained in some set $I_{M}$. In this case $f$ function approximation is carried out by $M$ vectors depending on its structure.

The error of approximation (2) is the sum of the remained coefficients $\varepsilon[M]=\left\|f-f_{M}\right\|^{2}=\sum_{(j, n) \notin I_{M}}\left|c_{j, n}\right|^{2}$.

Assuming that $e(t)=\sum_{(j, n) \notin I_{M}} c_{j, n} \Psi_{j, n}(t)$ component is a consequence of the noise factor influence, we receive representation of random time series in wavelet-space:

$$
f(t)=\sum_{(j, n) \in I_{M}} c_{j, n} \Psi_{j, n}(t)+e(t) .
$$

As a time series includes characteristic components and abnormal features, we will present it as follows:

$$
\begin{aligned}
& f(t)=\sum_{(j, n) \in I_{A}} a_{j, n} \Psi_{j, n}(t)+\sum_{(j, n) \in I_{D}} d_{j, n} \Psi_{j, n}(t)+e(t)= \\
& =f_{1}(t)+f_{2}(\mathrm{t})+\mathrm{e}(\mathrm{t})
\end{aligned}
$$

where $f_{1}(t)=\sum_{(j, n) \in I_{A}} a_{j, n} \Psi_{j, n}(t)$,

$$
f_{2}(t)=\sum_{(j, n) \in I_{D}} d_{j, n} \Psi_{j, n}(t),\left\{a_{j, n}\right\}_{(j, n) \in I_{A}}
$$

are set of approximating coefficients, describing characteristic features of data, $\left\{d_{j, n}\right\}_{(j, n) \in I_{D}}$ is set of the detailing coefficients describing abnormal features, $I_{A} \cup I_{D}=I_{M}$.

In $[14,24]$ demonstrated that absence of amplitude coefficients decrease when $j \rightarrow 0$, characterises presence of local features in $f(t)$ and operation of their detection can be realized on the basis of requirement check $\left|d_{j, n}\right| \geq T$, when $j \rightarrow 0$, where $T$ is some threshold value. Meantime, the least analyzed scale is limited by step of discrete time series sampling.

If wavelet $\Psi$ has compact carrier equal to $[-C, C]$, then assemblage of $(j, n)$ point pairs, such that some point $v$ is contained in $\Psi_{j, n}$ carrier, defines influence cone of point $v$ in scale-spatial plane [14]. As the 
carrier $\Psi_{j, n}$ on the scale $j$ is equal to

$$
\left\lfloor n-C * 2^{-j}, n+C * 2^{-j}\right\rfloor,
$$

then influence cone of point $v$ on the scale $j$ is defined by inequality:

$$
|n-v| \leq C * 2^{-j}, j=-1,-2, \ldots,-J .
$$

Let's consider that function $f$ in the neighbourhood of some point $v$ has abnormal feature of scale $j$, if in the neighbourhood of the point $v$ with the sizes defined by an influence cone, the condition is satisfied:

$$
\left|d_{j, n}\right| \geq T_{j}
$$

where $T_{j}$ is threshold value on scale $j$, time duration of abnormality is defined by the influence cone of point $v$.

Operation of scale $j$ abnormal features detection can be realized on the basis of threshold functions application

$$
P_{T_{j}}(x)=\left\{\begin{array}{l}
x, \text { если }|x| \geq T_{j} \\
0, \text { если }|x|<T_{j}
\end{array} .\right.
$$

The sets of detailing components $\left\{d_{j, n}\right\}_{(j, n) \in I_{D}}$ allocated in such a way define the component $f_{2}(t)$ of model Equation (3).

Intensity of abnormality on scale $j$ in point $v$ neighborhood we will define as $E_{f_{j, v}}=\max _{n}\left|d_{j, n}\right|$, where $n:|n-v| \leq C * 2^{-j}$.

Changes of intensity in time can be analyzed on the basis of value

$$
E_{f(t)}=\sum_{j}\left|c_{j, n}\right| \cdot
$$

The construction of wavelet-packages [14, 24] assumes recursive splitting of space $W_{j}: W_{j}=\oplus_{i=1}^{I} W_{j_{i}}^{p_{i}}$. Space $W_{j_{i}}^{p_{i}}$ admits orthonormalized basis

$$
\Psi_{j_{i}}^{p_{i}}=\left\{2^{j_{i} / 2} \Psi_{j_{i}}^{p_{i}}\left(2^{j_{i}} t-k\right)\right\}_{k \in Z} .
$$

Integration of corresponding basises of wavelet-packages $\left\{2^{j_{i} / 2} \Psi_{j_{i}}^{p_{i}}\left(2^{j_{i}} t-k\right)\right\}_{k \in Z, 1<i \leq I}$ defines orthonormalized basis $W_{j}$, that allows to restore function completely.

Detection and analysis of characteristic components of time series on the basis of wavelet-packages and neural networks joint application.

The Neural network creates mapping $y: f \rightarrow f^{\prime}$.

The set of weight coefficients of neuron input connections represents a column-vector [21]

$$
U=\left[u_{1}, \ldots, u_{N}\right]^{T},
$$

where $N$ is length of network input vector.

If $\hat{f}^{\prime}$ is a real network output, and $f^{\prime}$ is a desired one, then $f^{\prime}=y(f)$ is an unknown function, and a $\hat{f}^{\prime}=G(f, U)$ is its approximation which is reproduced by neural network. Procedure of network training is reduced to minimisation of approximation mean-square error on parameter $U$.

Giving to the input of the trained neural network values of function $f$ from an interval $[l-T+1, l]$, network becomes capable to compute anticipated function values on time interval $[l+1, l+\alpha]$, where $l$ is a current discrete moment of time; $\alpha$ is length of anticipation interval. The decision error is defined as difference between desired $f^{\prime}$ and real $\hat{f}^{\prime}$ output values during the discrete time moment $l$.

The error vector is the vector where $i$ element is

$$
\varepsilon_{i}(l)=\hat{f}_{i}^{\prime}(l)-f^{\prime}{ }_{i}(l),
$$

where $l$ is a current time moment, $i$ is a current position on anticipation interval.

Algorithm of training and control sets formation:

1. An initial data array $\{f(k)\}_{k=1}^{K}$, where $K$ is a sampling length, is divided on $L$ blocks $Q:\{f(k)\}_{k=1}^{K}=\left(\{f(k)\}_{k=1}^{Q},\{f(k)\}_{k=2}^{Q+1}, \ldots,\{f(k)\}_{k=K-Q}^{K}\right)$ long.

2. On the basis of wavelet-packages construction, for each block $s$ we have representation $f$ in the form of a linear combination different-scale components: $f^{s}=f_{1}^{s}+f_{2}^{s}+\ldots+f_{p}^{s}$, where every component $f_{i}^{s}=\sum_{\left(j_{i}, k\right) \in I_{s}^{p_{i}}} \beta_{j_{i}, k}^{s} \Psi_{j_{i}}^{p_{i}}, \Psi_{j_{i}}^{p_{i}} \in W_{j_{i}}^{p_{i}}$ in wavelet-space is uniquely defined by coefficients sequence $\bar{\beta}_{j_{i}}^{s}=\left\{\beta_{j_{i}, k}^{s}\right\}_{\left(j_{i}, k\right) \in I_{s}^{p_{i}}}, \quad \beta_{j_{i}, k}^{s}=\left\langle f^{s}, \Psi_{j_{i}}^{p_{i}}\right\rangle, \quad W_{j_{i}}^{p_{i}}$ are wavelet-package spaces.

3. Every detected component defines a subspace of time series features space. As $W_{j}^{p_{i}}$ are wavelet-packages spaces, then is obtained: $\bigcap_{i=1}^{I} W_{i}^{p_{i}}=\{0\}, \bigcup_{i=1}^{I} W_{i}^{p_{i}}=V$.

Thus, for each unit $S$ separation of data features in space is received Figure 1. Using the following sets of detected features

$$
\begin{aligned}
& \left\{f_{j_{1}, p_{1}}^{s}\right\}_{s=\overline{1, L}} ;\left\{f_{j_{1}, p_{2}}^{s}\right\}_{s=\overline{1, L}} \quad ; \ldots ;\left\{f_{j_{1}, p_{I}}^{s}\right\}_{s=\overline{1, L}} ; \ldots ; \\
& \left\{f_{j_{I}, p_{I}}^{s}\right\}_{s=\overline{1, L}} ;\left\{f_{j_{1}, p_{1}}^{s}, f_{j_{1}, p_{2}}^{s}\right\}_{s=\overline{1, L}} ; \ldots ;\left\{f_{j_{I}, p_{1}}^{s}, f_{j_{I}, p_{2}}^{s}\right\}_{s=\overline{1, L}} ;
\end{aligned}
$$


$\left\{f_{j_{1}, p_{1}}^{s}, f_{j_{1}, p_{2}}^{s}, f_{j_{1}, p_{3}}^{s}\right\}_{s=\overline{1, L}} ; \ldots\left\{f_{j_{1}, p_{1}}^{s}, f_{j_{1}, p_{2}}^{s}, \ldots, f_{j_{1}, p_{1}}^{s}\right\}_{s=\overline{1, L}}$ $; \ldots ;\left\{f_{j_{I}, p_{1}}^{s}, f_{j_{I}, p_{2}}^{s}, \ldots, f_{j_{I}, p_{I}}^{s}\right\}_{s=1, L}$ we form training and control sets for neural networks.

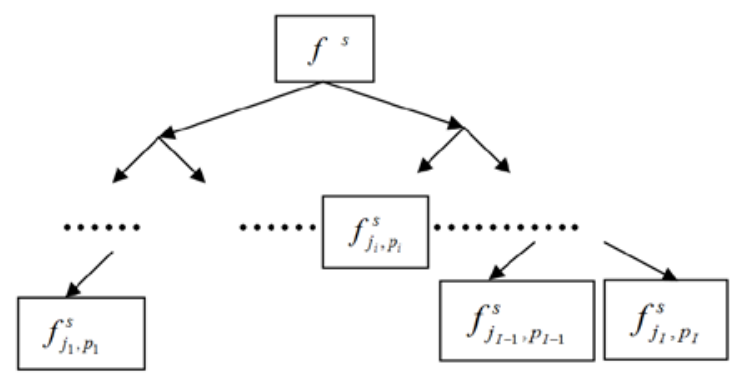

Figure 1. The scheme of data separation in wavelet-images space.

\section{Algorithm of "the best" network construction:}

Step 1: Carry out wavelet-restoration of a component $f_{j_{1}, p_{1}}^{s}$ for each data unit $s$ and form training set on the basis of combinations of the restored data from various units. Construct network 1 of variable structure [21] (variable structure network is a multilayer feed forward network, which architecture is defined by minimisation of decision error on training vectors set), train and test it.

Step 2: Carry out wavelet-restoration of components $f_{j_{I}, p_{I}}^{s}$ for each data unit $s$ and form training set on the basis of combinations of the restored data from various units. Construct a network 2 of variable structure, train and test it.

Etc.

Step r: Carry out wavelet-restoration of components $f_{j_{I}, p_{1}}^{s}, f_{j_{I}, p_{2}}^{s}, \ldots, f_{j_{I}, p_{I}}^{s}$ for each data unit $s$ and form training set on the basis of combinations of the restored data from various units. Construct network $r$ of variable structure, train and test it.

On the basis of the analysis of results of received neural networks operation the "best" network is defined: "the best" is considered to be the network having the least decision error on test set

$$
E_{\mathrm{M}, \min }=\min _{\alpha}\left(\frac{1}{\mathrm{M}} \sum_{l=1}^{\mathrm{M}} \sum_{i=1}^{f} \varepsilon_{i}(l)^{2}\right),
$$

where $\alpha$ is a neural network number, $\alpha=\overline{1, r}, \mathrm{M}$ is a length of analyzed network output vector, $f$ is a length of an anticipation interval.

The training data set is a set of observations containing features of studied process. On the basis of waveletpackages construction, we have data features separation in space. During training and designing each network learns a subset of input data features and approximates them. "The best" network is the network having the least decision error on test set. Therefore data subset used at training of the "best" network will contain the most typical features of studied process. In wavelet-space this subset is represented by set of coefficients $\left\{a_{j, n}\right\}_{(j, n) \in I_{A}}$, defining component $f_{1}(t)$ of time series model Equation (3).

If there is an abnormal feature in the data, then a change of their structure occurs. Therefore operation of abnormal features detection on the basis of a neural network can be constructed by processing and analysis of decision errors $\varepsilon_{i}$ : if

$$
E_{Z}=\frac{1}{Z} \sum_{l=1}^{Z} \sum_{i=1}^{f} \varepsilon_{i}(l)^{2} \geq \mathrm{P},
$$

where $Z$ is an observation frame length, $P$ is a beforehand specified threshold value, then within an analyzed time frame we have abnormality.

\section{Results of experiments}

In experiments $f_{\mathrm{O}} \mathrm{F} 2$ data were used, received by automatic ionospheric station located in Paratunka settlement, Kamchatka peninsula. Data recording occurs once an hour. For experiments results of $\mathrm{f}_{\mathrm{O}} \mathrm{F} 2$ measurements dated 1979 - 2011 were taken. In the process of analysis, data of the Earth magnetic field (H-component) were used to define magnetospheric disturbances degree, characterising Solar activity. As basic functions the class of Daubechies orthogonal wavelets: db2, db3, db4 was used.

Following the results obtained in [3], for detection of abnormalities on the basis of operation Equation (4) were used the threshold values defined in the process of algorithm operation by formula:

$$
\begin{aligned}
T_{j} & =\underset{n, n=\overline{1, V}}{\operatorname{med}}\left(\left|d_{j, n}\right|\right)+\theta * S t_{j}
\end{aligned}
$$

value defined within the analyzed sliding time frame of length $V, V=168$ readouts, med is a median defined within the analyzed sliding time frame of length $V$. The coefficient $\theta=3$ has been defined statistically. The detected time-and-frequency intervals containing abnormal features, are shown on Figure 2-5 (b) by shades of grey colour. Ionospheric disturbances intensity changes in time were analyzed on the basis of value Equation (5), Figure 2-5 (c).

On the basis of described above algorithms training and control sets for neural networks have been generated and "the best" network consisting of three layers that 
allows to perform forecast of the $\mathrm{f}_{\mathrm{O}} \mathrm{F} 2$ data with anticipation step equal to 3 hours has been constructed. Detected on the basis of "the best" network characteristic component of $\mathrm{f}_{\mathrm{O}} \mathrm{F} 2$ time series looks like follows:

$$
f_{1}(t)=\sum_{j_{i}, k} a_{j_{i}, k} \Psi_{j_{i}}^{p_{i}}, \Psi_{j_{i}}^{p_{i}} \in W_{j_{i}}^{p_{i}}, \quad j_{i}=3, p_{i}=1,
$$

$k \in Z$.

The analysis of neural networks decision errors (Equations (6), (7)) has shown that Daubechies basis function of an order 3 provides the least $\mathrm{f}_{\mathrm{O}} \mathrm{F} 2$ data approximation error for the analyzed time periods. The analysis Figure 2-5 (a) shows that during the periods of increasing seismic activity, neural network error increase, characterising presence of abnormal features in the data is observed. The abnormalities detected on the basis of discrete wavelet-transformation (Equation (4), Figure 2-5 (b)) also prove this result. The detailed analysis of abnormalities shows that they are non-uniformly distributed both in time and on scales and characterised by various intensity (value $E_{f(t)}$, Figure 2-5 (c)).

Comparison of the received results with the Earth magnetic field data Figure 2-5 (d) shows that analyzed litospheric processes in most cases are observed against increased solar activity.

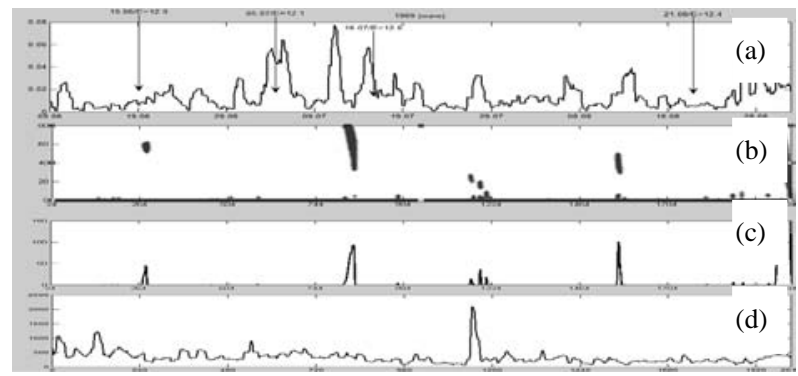

Figure 2. Results of $f_{0} F 2$ data processing 1969: (a) a vector of a neural network error; (b) the time-and- frequency intervals containing abnormal features; (c) intensity of abnormalities; (d) H-component of the Earth magnetic field. Arrows note the moments of earthquakes occurrence.

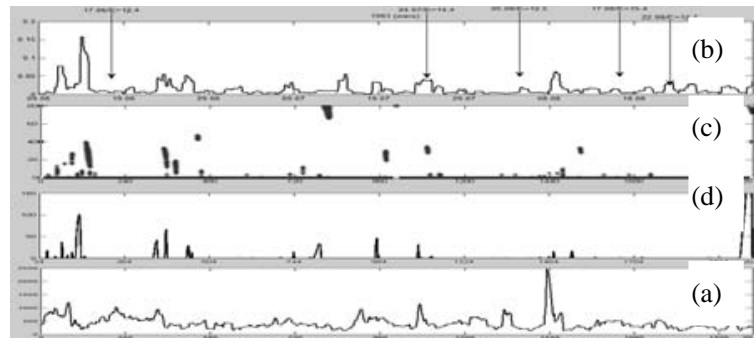

Figure 3. Results of $f_{0} F 2$ data processing 1983: (a) a vector of a neural network error; (b) the time-and-frequency intervals containing abnormal features; (c) intensity of abnormalities; (d) H-component of the Earth magnetic field. Arrows note the moments of earthquakes occurrence.

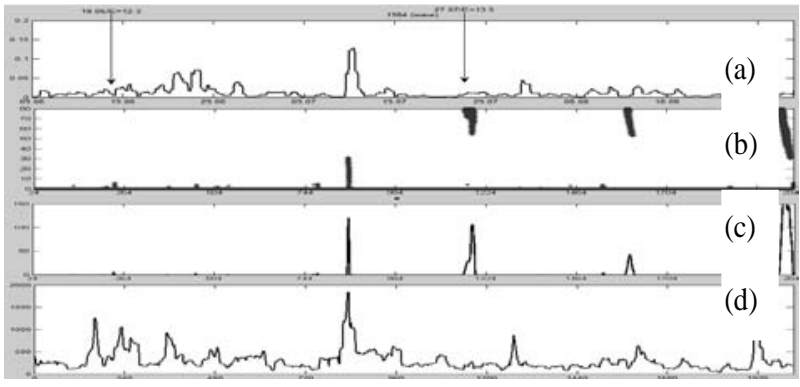

Figure 4. Results of $f_{0} F 2$ data processing 1984: (a) a vector of a neural network error; (b) the time-and-frequency intervals containing abnormal features; (c) intensity of abnormalities; (d) H-component of the Earth magnetic field. Arrows note the moments of earthquakes occurrence.

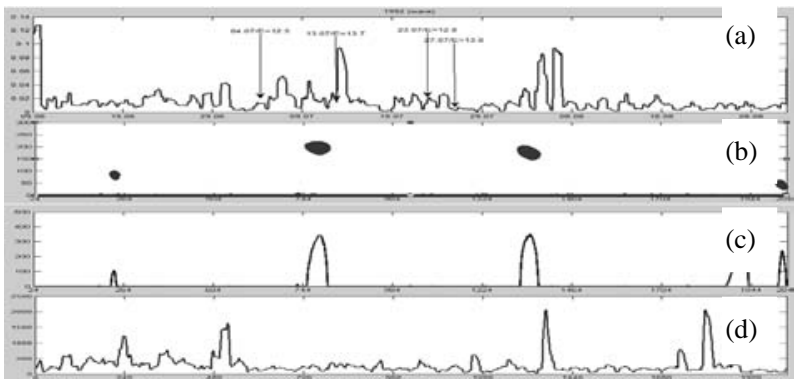

Figure 5. Results of $f_{\mathrm{O}} F 2$ data processing 1992: (a) a vector of a neural network error; (b) the time-and-frequency intervals containing abnormal features; (c) intensity of abnormalities; (d) H-component of the Earth magnetic field. Arrows note the moments of earthquakes occurrence.

\section{Conclusions}

On an example of the $\mathrm{f}_{\mathrm{O}} \mathrm{F} 2$ data for studying of time features of ionosphere parametres and detection of abnormalities arising during the periods of increased solar or seismic activity, the method based on combination of wavelet-transformation and neural networks is offered. Automatic algorithms of detection and analysis of characteristic components of $\mathrm{f}_{\mathrm{O}} \mathrm{F} 2$ series are developed.

Method approbation on the data received by automatic ionospheric station Paratunka settlement Kamchatka peninsula, has proved its efficiency and has allowed to detect the abnormal features arising during the periods of solar activity increasing and on the eve of catastrophic earthquakes on Kamchatka. The detected characteristic components of $\mathrm{f}_{\mathrm{O}} \mathrm{F} 2$ series have allowed to analyse ionospheric parametres variations during the summer period of time and their essential change during the periods of seismic and solar activity increasing. The detailed analysis of the allocated abnormal features has shown that during the periods of seismic or solar activity increasing in variations of $\mathrm{f}_{\mathrm{O}} \mathrm{F} 2$ series local differentscale periodicities having non-uniform distribution both on time and on scales arise. 


\section{Acknowledgements}

The present paper and the research are supported by the grant of the President of the Russian Federation MD-2199.2011.9, the grant of the Russian Foundation for Basic Research (Far Eastern Branch of the Russian Academy of Sciences, Russia) № 11-07-98514-r-vostok_a and the grant «U.M.N.I.K.» - 8283r/10269 dated 6/30/2010.

Data of the seismic catalogue is kindly given by the Kamchatka branch of geophysical service of the Russian Academy of Sciences (Petropavlovsk-Kamchatskiy).

\section{REFERENCES}

[1] M.G. Dyomin, "Ionosphere of the Earth. Plasma Heliophysics,” PhysMathlit, Moscow, 2008.

[2] V.V. Bogdanov, V.V. Geppener and O.V. Mandrikova, "Modeling of Non-Stationary Time Series of Geophysical Parameters with a Complex Structure," St.-Petersburg, 2006.

[3] O. V Mandrikova, Yu.A. Polozov and A.S. Perezhogin, "Wavelet-Technology of the Ionospheric Data Analysis," Scientific Notes of the Belgorod State University, a Series "History. Political Science. Economy. Computer Science", Belgorod, No. 19, 2011, pp. 113-118.

[4] O. V Mandrikova and Yu.A. Polozov, "Criteria of Wavelet-Function Choice in Problems of Approximation Natural Time Series of Complex Structure," Information Technology, Moscow, No. 1, 2012, pp. 31 - 36.

[5] O. V. Mandrikova, "Multicomponent Model of a Signal with Complex Structure," Problems of Evolution of Open Systems, Issue 10, Vol. 2, 2008, pp.161-172.

[6] Yu. A. Polozov, "Method of Training Set Formation for a Neural Network on the Basis of a Wavelet-Filtration," News of High Schools, the North Caucasian Region, a Series Natural Sciences, No. 3, 2010, pp. 12-16.

[7] E.V. Liperovskaja, V. A. Liperovsky and O. A. Pokhotelov, "About Disturbances in Ionosphere F-Area before Earthquakes,” Geophysical researches, No. 6, 2006, pp. 51-58.

[8] A. A. Namgaladze, "The Physical Mechanism and Mathematical Modeling of Ionospheris Harbingers of the Earthquakes Recorded in Full Electronic Maintenance,” Geomagnetism and Aeronomia, Vol. 49, No. 2, -2009, pp. 267-277.

[9] O. V. Mandrikova, Yu. A. Polozov and T. L. Zaliaev, "Methods of Analysis and Interpretation of Ionospheric Critical Frequency $\mathrm{F}_{\mathrm{O}} \mathrm{f}$ Data Based on Wavelet Transform and Neural Networks," European Seismological Commission 33-rd General Assembly (GA ESC 2012), 19-24 August 2012, Thesis, Moscow.

[10] Y. Polozov, O. Mandrikova and V. Bogdanov, "Detection of Anomalies in Ionospheric Increased Seismic Activity," International Union of Geodesy and Geophysics (IUGG), 28 June - 07 July 2011, Thesis, Melbourne.

[11] O. V Mandrikova, Yu. A. Polozov, N.V. Glushkova and T.L. Zalyaev, "Technology of Allocation of Anomalies in Ionospheric Data on the Basis of Combination
Wavelet-Transformation and Neural Networks," International Conference "Intelligent Information Processing» (IIP-2012), Montenegro, Budva,16-22 September 2012, pp. 524-527.

[12] O. V Mandrikova and Yu. A. Polozov, "Method of Allocation and Classification of Anomalies in Ionospheric Parameters on the Basis of Combination of Wavelet-Transformation and Neural Networks," 14 International Conference «Digital Signal Procssing» (DSPA - 2012), Moscow, 28-30 March 2012, pp. 353-356.

[13] O.V. Mandrikova, 'Optimization of a Neural Network Training Procession on The Basis of Application of a Design of Wavelet-Transformation (on an Example of Modeling Ionospheric Signal Representation)," Automation and Modern Technologies, No. 3, 2009, pp.14-17.

[14] S. Mallat, “A Wavelet Tour of Signal Processing,” World, Moscow, 2005.

[15] O. V. Mandrikova, I. S. Solovjev, V. V. Geppener and D. M. Klionskiy, "Analyzing Subtle Features of Natural Time Series by Means of a Wavelet-Based Approach," Pattern Recognition and Image Analysis, Vol. 22, No. 2, 2012, pp. 323-332. doi:10.1134/S1054661812020083

[16] O.V. Mandrikova , I.S. Solovyev, V.V.Geppener, D.M. Klionskiy and R. T. Al-Kasasbeh, "Analysis of the Earth's magnetic field variations on the basis of a wavelet-based approach,” Digital Signal Processing, In Press.

[17] C. K. Chui and J. Z. Wang, "A general framework of compactly supported splines and wavelets,” CAT Report 219, Texas A\&M Univ., 1990.

[18] Ali G. Hafez and Essam Ghamry, "Automatic detection of geomagnetic sudden Commencements via Time Frequency Clusters,” Advances Space Research, Vol. 48, Issue 9, 2011, pp. 1537-1544. doi:10.1016/j.asr.2011.05.025

[19] Ali G. Hafez, Tahir A. Khan and T. Kohda, “Clear P-wave Arrival of Weak Events and Automatic Onset Determination Using Wavelet Filter Banks," Digital Signal Processing, Vol. 20, Issue 3, 2010, pp. 715-723. doi:10.1016/j.dsp.2009.10.002

[20] D. B. Percival and A. T. Walden, "Wavelet Methods for Time Series Analysis,” (Cambridge Series in Statistical and Probabilistic Mathematics), Cambridge University Press, 2000.

[21] A.D. Ageev, "Neuromatematika: Studies. The Grant for High Schools,” IPRJR, Moscow, 2002.

[22] H.A. Barhatov and C.E. Revunov, "Forecasting of Critical Frequency Ionospheric Layer F2 a Method of Artificial Neural Networks," VIII Nizhniy Novgorod Session of Young Scientists. Natural-Science Disciplines, Thesis, Dzerzhinsk, 2003.

[23] H.A. Barhatov, C.E. Revunov and V.P. Urjadov, "Technology of Artificial Neural Networks for Forecasting of Critical Frequency Ionospheric Layer F2," News of HIGH SCHOOLS "Radio Physicists", Vol. 48, 2005, pp.1-15.

[24] I. Daubechies, “Ten Lectures on Wavelets,” «Regular and Chaotic Dynamics», Izhevsk, 2001 